\title{
REACTION AND MASS TRANSFER IN A MACRORETICULAR RESIN CATALYST
}

\author{
SON-KI IHM**, SUnG-SUP SUH, AND IN-HWAN OH \\ The Korea Advanced Institute of Science and Technology, \\ P.O. Box 150, Chongyangni, Seoul 131 Korea
}

\begin{abstract}
A model is proposed for a macroreticular resin catalyst to give the overall effectiveness factor in terms of the micro- and macro-effectiveness factors and the fraction of the surface layer active sites. Different values of the fraction can represent some possible cases of porous catalysts; i.e., a single or uniform pore and a bidisperse pore structure.
\end{abstract}

\section{Introduction}

Most heterogeneous catalysts are made by pelletizing active powders and show a bidisperse pore structure of micro- and macropores. Various models ${ }^{11}$ have been proposed to explain diffusion and reaction in such porous catalysts. Macroreticular ion exchange resins, which consist of very small randomly packed gelular microparticles with continuous nongel pores, might be analyzed in a similar manner by taking the microparticles as having micropores.

Mingle and Smith $^{4}$, Carberry ${ }^{2}$, and Örs and Dogu $^{6)}$ have derived expressions for the micro- and macro-effectiveness factor. In their calculations the resulting overall effectiveness factor was essentially the product of the micro- and macro-effectiveness factors and the contribution from macro-pore walls to reaction is assumed to be negligible. Tartarelli et al..$^{8)}$ analyzed a second-order reaction by introducing a generalized Thiele modulus and calculated the effectiveness factor for an isothermal condition by a numerical method.

For resin catalysis, Frisch ${ }^{33}$ proposed a model with radially oriented cylindrical macropores in the gel structure and considered diffusion-limited first-order reversible reactions catalyzed by macroreticular ion exchange resins in which both macropore and matrix diffusion in the polymer were taken into account. Ruckenstein et al. ${ }^{7)}$ developed a similar macro-micro pore model for transient sorption in solids having bidisperse pore structures which showed the influence of the competing effects of macropore and micropore diffusion.

From the viewpoint of the scanning electron micrograph the cylindrical geometry of the macropore may not be adequate for describing the macro-

Received August 31, 1981. Correspondence concerning this article should be addressed to $\mathrm{S}$. Ihm. reticular ion exchange resin catalyst. The present study proposes a more generalized model which would be of aid in understanding the intraparticle mass transfer and its interaction with reaction, especially for macroreticular resins.

\section{A Model for the Macroreticular Ion Exchange Resin}

A macroreticular ion exchange resin particle is composed of spherical microparticles with free space between them, which accounts for the porosity of the resin. Therefore, the macroreticular resin can be envisaged as two phases, i.e., microspheres of uniform size and pores formed by the space between the microspheres. It is assumed that the radius of the microspheres is uniform and much smaller than that of the resin particle.

Two different kinds of active sites exist in the microsphere of macroreticular resin. The fraction $\gamma$ of total active sites are located on the surface of the microparticle, providing easy access for the reactants. The reaction can proceed on these external active groups without being preceded by permeation into the micropatricle. However, the remaining functional groups of fraction $1-\gamma$ are located within the polymer matrix of the microparticle. The molecules from the external phase must penetrate through the polymer matrix in order to gain access to the inner active functional groups.

The external active groups and a large surface area due to high crosslinking are the characteristics of macroreticular resins, which can catalyze reactions even among nonpolar molecules while gelular resins cannot. The active acidic sites are uniformly distributed over the pore walls (surface of the microsphere) and also in the inner gelular phase of the microsphere. Reaction occurs on the pore walls as well as inside the gelular microspheres. A schema- 
tic diagram of a macroreticular resin catalyst is shown in Fig. 1.

\section{Governing Equations}

For the pore space, diffusional flux is balanced by disappearance due to reaction on the walls and diffusion into the microspheres, and for the microsphere diffusional flux is balanced by reaction only.

By defining the dimensionless variables as

$$
y_{a}=\frac{C_{a}}{C_{o}}, y_{i}=\frac{C_{i}}{C_{0}}, x_{a}=\frac{r_{a}}{R_{a}}, x_{i}=\frac{r_{i}}{R_{i}}
$$

and the parameters as

$$
\begin{aligned}
& m_{i}=R_{i} \sqrt{\frac{(\overline{1-\gamma}) \bar{k} \overline{C_{o}^{n-1}}}{n^{\prime} \overline{V_{i} D_{i}}}} \\
& m_{a}=R_{a} \sqrt{\frac{e k \overline{C_{o}^{n-1}}}{V_{a} D_{a} \varepsilon}}
\end{aligned}
$$

the governing equations at steady state can be written for an $n$-th order irreversible reaction for the pore space

$$
\frac{d^{2} y_{a}}{d x_{a}^{2}}+\frac{2}{x_{a}} \frac{d y_{a}}{d x_{a}}=\gamma m_{a}^{2} y_{a}^{n}+3(1-\gamma) \frac{m_{a}^{2}}{m_{i}^{2}}\left(\frac{d y_{i}}{d x_{i}}\right)_{x_{i}=1}
$$

and for the microsphere

$$
\frac{d^{2} y_{i}}{d x_{i}^{2}}+\frac{2}{x_{i}}-\frac{d y_{i}}{d x_{i}}=m_{i}^{2} y_{i}^{n}
$$

and the boundary conditions are

$$
\begin{aligned}
& x_{a}=0 ; d y_{a} / d x_{a}=0 \\
& x_{a}=1 ; y_{a}=1 \\
& x_{i}=0 ; d y_{i} / d x_{i}=0 \\
& x_{i}=1 ; y_{i}=y_{a}\left(x_{a}\right)
\end{aligned}
$$

where parameter $m_{i}$ represents the Thiele modulus for a microsphere and $m_{a}$ the Thiele modulus for the pore space. If $m_{t}$ is defined as the ratio of the time scales for the processes taking place in the resin pore and in the microparticle respectively,

$$
m_{t}=\frac{t_{a}}{t_{i}}=\frac{(1-\varepsilon) D_{i} / R_{i}^{2}}{\varepsilon D_{a} / R_{a}^{2}}=(1-\gamma) \frac{m_{a}^{2}}{m_{i}^{2}}
$$

where $t_{a}$ represents the characteristic time required for diffusion through the pore of the resin particle and $t_{i}$ the characteristic time required for permeating through the microparticle, Eq. (4) becomes

$$
\frac{d^{2} y_{a}}{d x_{a}^{2}}+\frac{2}{x_{a}} \frac{d y_{a}}{d x_{a}}=\frac{m_{i}^{2} m_{t} \gamma}{1-\gamma} y_{a}^{n}+3 m_{t}\left(\frac{d y_{i}}{d x_{i}}\right)_{x_{i}=1}
$$

The microeffectiveness factor is defined as

$$
\eta_{i}=\frac{3}{m_{i}^{2} y_{a}^{n}}\left(\frac{d y_{i}}{d x_{i}}\right)_{x_{i}=1}
$$

Introducing Eq. (9) into Eq. (4) results in a more general form, or

$$
\frac{d^{2} y_{a}}{d x_{a}^{2}}+\frac{2}{x_{a}} \frac{d y_{a}}{d x_{a}}=m_{a}^{2}\left[\gamma+(1-\gamma) \eta_{i}\right] y_{a}^{n}
$$

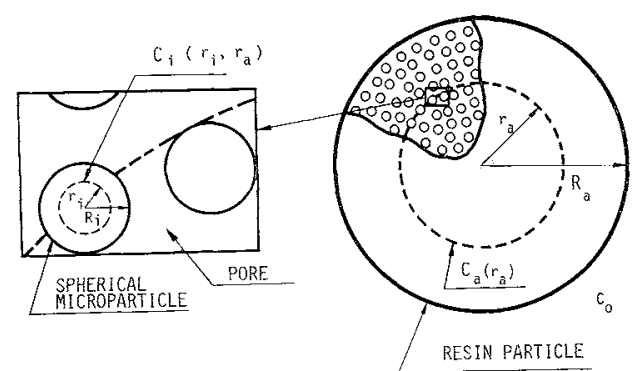

Fig. 1 Schematic diagram of a resin particle

Before solving Eqs. (5) and (10) it can be shown that the overall effectiveness factor $\eta_{0 v}$ is expressed in terms of the average micro-effectiveness factor $\bar{\eta}_{i}$, the macro-effectiveness factor $\eta_{a}$ and the fraction $\gamma$ of active sites located on the pore walls as follows:

$$
\begin{gathered}
\eta_{o v}=\frac{3}{m_{a}^{2}}\left(\frac{d y_{a}}{d x_{a}}\right)_{x_{a}=1} \\
=\left[(1-\gamma) \bar{\eta}_{i}+\gamma\right] \eta_{a} \\
\bar{\eta}_{i}=\int_{0}^{1} x_{a}^{2} y_{a}^{n} \eta_{i} d x_{a} / \int_{0}^{1} x_{a}^{2} y_{a}^{n} d x_{a}
\end{gathered}
$$

and

$$
\eta_{a}=\frac{3}{M^{2}}\left(\frac{d y_{a}}{d x_{a}}\right)_{x_{a}=1}
$$

where $M$ is the modified Thiele modulus defined as

$$
M^{2}=m_{a}^{2}\left[\gamma+(1-\gamma) \bar{y}_{i}\right]
$$

$\bar{\eta}_{i}$ is the averaged value of the local effectiveness factor $\eta_{i}$ for a microsphere which, except for a firstorder reaction, depends upon the radial position of the resin particle as the concentration in the pore space varies along the radial position.

\section{Results and Discussion}

\section{1. First-order reaction}

For a first-order reaction $(n=1)$, the analytical solutions of Eqs. (5) and (10) can easily be found, or

$$
\begin{aligned}
& y_{a}=\frac{1}{x_{a}} \sinh M x_{a} \\
& \sinh M \\
& y_{i}=\frac{y_{a}}{x_{i}} \sinh m_{i} x_{i} \\
& \sinh m_{i}
\end{aligned}
$$

Effectiveness factors are given as

$$
\begin{aligned}
& \bar{\eta}_{i}=\eta_{i}=\frac{3}{m_{i}}\left(\frac{1}{\tanh m_{i}}-\frac{1}{m_{i}}\right) \\
& \eta_{a}=\frac{3}{M}\left(\frac{1}{\tanh M}-\frac{1}{M}\right)
\end{aligned}
$$

and

$$
\begin{aligned}
\eta_{o v} & =\frac{3}{m_{a}^{2}}\left(\frac{M}{\tanh M}-1\right) \\
& =\left[(1-\gamma) \frac{3}{m_{i}}\left(\frac{1}{\tanh m_{i}}-\frac{1}{m_{i}}\right)+\gamma\right] \frac{3}{M}\left(\frac{1}{\tanh M}-\frac{1}{M}\right)
\end{aligned}
$$




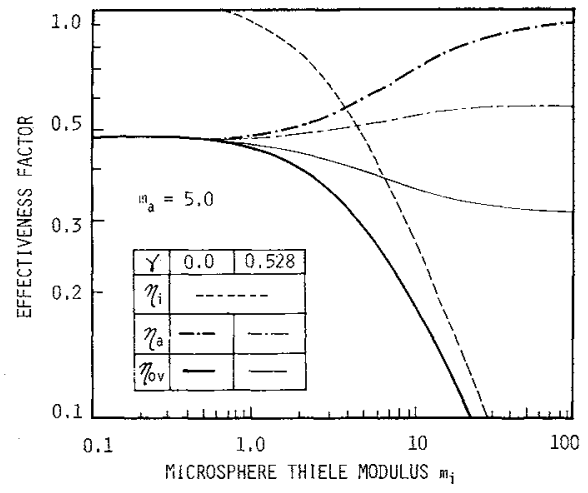

Fig. 2 Effectiveness factors for 1st-order reaction

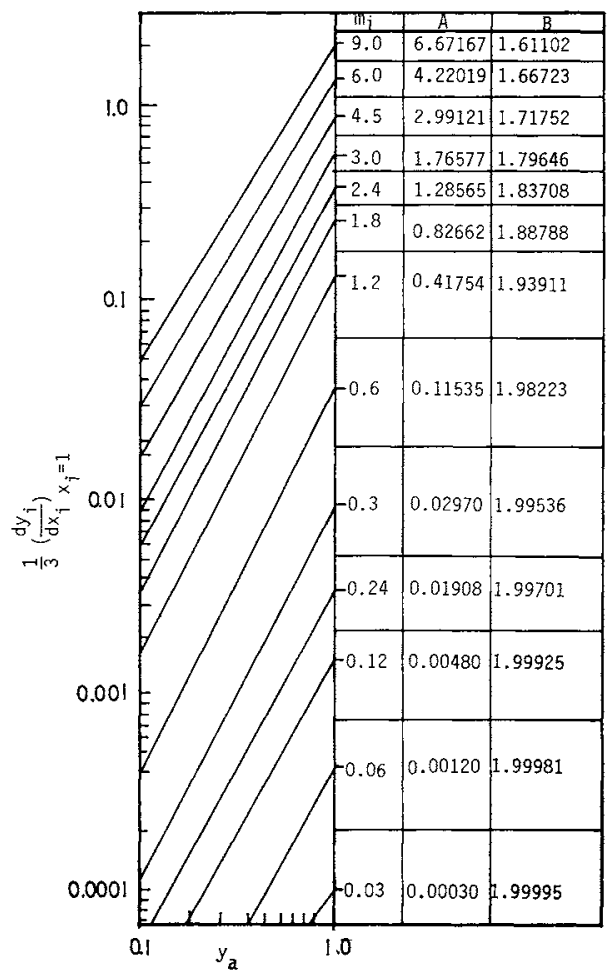

Fig. 3 Concentration gradient as a function of of $m_{i}$ and $y_{a}$

Figure 2 shows these effectiveness factors, $\eta_{i}, \eta_{a}$ and $\eta_{o v}$ for the case of $\gamma=0$ and $\gamma=0.528$ when $m_{a}=5.0$. As permeation becomes more difficult $\left(m_{i} \sim \infty\right)$, the micro-effectiveness factor $\eta_{i}$ approaches zero and as the reaction controls the rate process $\left(m_{i} \sim 0\right), \eta_{i}$ becomes unity. It should be noted that the microeffectiveness factor $\eta_{t}$ is the same as the effectiveness factor of a uniformly porous catalyst particle with a Thiele modulus $m_{i}$.

\section{2 Second-order reaction}

For a second-order reaction $(n=2)$, analytical solutions cannot be found and numerical integrations were performed by the fourth-order Runge-Kutta method with varying step size to solve the nonlinear boundary value problem.
From Eq. (5), $\left(d y_{i} / d x_{i}\right)_{x_{i}=1}$ can be approximated as a function of $m_{i}$ and $y_{a}$ to be introduced to Eq. (4).

$$
\left(\frac{d y_{i}}{d x_{i}}\right)_{x_{i}=1}=\left[\begin{array}{ll}
\frac{1}{3} m_{i}^{2} y_{a}^{2} & \left(m_{i} \ll 1\right) \\
A y_{a}^{B} & (\text { intermediate }) \\
\sqrt{ } \overline{2} / \sqrt{3} m_{i} y_{a}^{3 / 2} & (m \gg 1)
\end{array}\right.
$$

and the micro-effectiveness factor becomes

$$
\eta_{i}=\left[\begin{array}{ll}
1 & \left(m_{i} \ll 1\right) \\
\frac{3}{m_{i}^{2}} A y_{a}^{B-2} & \text { (intermediate) } \\
\sqrt{ } 6 \frac{1}{m_{i} y_{a}^{1 / 2}} & \left(m_{i} \gg 1\right)
\end{array}\right.
$$

Equation (21a) is derived from Eq. (9) because $\eta_{i}$ becomes unity for $m_{i} \ll 1$, and Eq. (21c) can be derived from the asymptotic solution for $m_{i} \gg 1$.

By solving Eq. (5) for a given value of $m_{i}$, the concentration gradients at the external surface of the microsphere $\left(d y_{i} / d x_{i}\right)_{s_{i}=1}$ can be obtained. The $\log -\log$ plot of $\left(d y_{i} / d x_{i}\right)_{x_{i}=1}$ versus $y_{a}$ was found to give a straight line, as shown in Fig. 3, confirming the validity of the approximation by Eq. (21b), and the values of $\mathrm{A}$ and $\mathrm{B}$ obtained for the different values of $m_{i}$ are also listed.

With Eqs. (21) or (22), Eq. (4) or (10) can be solved numerically and the concentration profile can be obtained at given values of $\gamma, m_{i}$, and $m_{\alpha}$. Accordingly, the averaged micro-effectiveness factors are evaluated from the concentration profile. That is,

$$
\bar{\eta}_{i}=\left(\begin{array}{ll}
1 & \\
\frac{3 A}{m_{i}^{2}} \int_{0}^{1} x_{a}^{2} y_{a}^{B} d x_{a} & \left(m_{i} \ll 1\right) \\
\int_{0}^{1} x_{a}^{2} y_{a}^{2} d x_{a} & \text { (intermediate) } \\
\frac{\sqrt{6}}{m_{i}} \int_{0}^{1} x_{a}^{2} y_{a}^{3 / 2} d x_{a} & \\
\int_{0}^{1} x_{a}^{2} y_{a}^{2} d x_{a} & \left(m_{i} \gg 1\right)
\end{array}\right.
$$

The effectiveness factors for the cases of $\gamma=0$ and $\gamma=0.528$ are plotted in Figs. 4 and $\mathbf{5}$ respectively in terms of $m_{a}$, and in Fig. 6 in terms of $m_{t}$ and $m_{i}$.

As the microsphere Thiele modulus $m_{i}$ increases, the diffusion rate into the microsphere becomes slow and the reactants tend to accumulate in the pore space. The uniform concentration profile in the pore results in the enhancement of the macro-effectiveness factor $\eta_{a}$. However, the overall effectiveness factor will decrease due to diffusion limitations in the microspheres.

If very few active sites are located on the pore walls (i.e., $\gamma=0$ ), there is little reaction on the pore walls. However, if a large portion of active sites is located on the pore walls (i.e., $\gamma \neq 0$ ), they play the role of sink for reactants in the pore space due to the wall reaction. Hence the overall effectiveness factor for 
the case of $\gamma \neq 0$ is different from that for the case of $\gamma=0$ showing a different asymptote.

If the diffusion resistance through the microsphere is negligible (or microsphere Thiele modulus $m_{i}$ becomes smaller) reaction occurs in the entire range of the microsphere and the overall process depends only on diffusion through the pore space. Therefore, the overall effectiveness factor $\eta_{o v}$ becomes a function of the Thiele modulus $m_{a}$ alone.

Figure 6 shows that as $m_{t}$ increases, the diffusion rate of the reactant molecules into the pore of the resin particle decreases and the overall effectiveness factor decreases.

\section{3 Limiting cases of $\gamma=0$ and $\gamma=1$}

If $\gamma=0$, the present model is the same as the bidisperse porous catalyst models, and the effectiveness factor becomes simply the product of the micro- and macro-effectiveness factor, or

$$
\eta_{o v}=\bar{\eta}_{i} \eta_{a}
$$

The same relation can be obtained from the model proposed by Örs and Dogu ${ }^{6)}$ and also by Carberry ${ }^{2}$.

If $\gamma=1$, the governing equation reduces to that for a single pore or uniformly porous catalyst, for which numerous studies ${ }^{1)}$ can be referenced.

It is the merit of the present model that three possibilities in typical heterogeneous catalysis, i.e. a single pore, a biporous catalyst and an ion exchange resin catalyst, can be explained by a single expression.

\section{Experimental Examples-Temperature Effect}

As an illustration, some experimental results obtained by the authors are explained in the following:

A) The liquid-phase esterification of phenol and acetic acid was performed in a batch reactor using macroreticular ion exchange resin catalysts (Amberlyst 15 and Amberlyst XN-1010) and experimental data on the initial reaction rate were analyzed by the present model ${ }^{8}$. Under the experimental condition of $\eta_{a}=1$ (i.e., when the variations in size of resin particle do not affect the overall rate), the overall initial reaction rate $r_{o v}$ could be expressed as

$$
r_{o v}=r_{x} \eta_{o v}=r_{x}\left[\gamma+(1-\gamma) \bar{\eta}_{i}\right]
$$

In the range of low reaction temperature most reaction occurs on the surface layer of the microparticle (the pore walls) because the reactant cannot easily penetrate into the highly crosslinked gel phase of the microparticle. Therefore, micro-effectiveness factor $\bar{\eta}_{i}$ becomes very small. As the reaction temperature increases the gel phase can participate in the reaction due to increased swellability and this results in higher micro-effectiveness factor. In the extreme case of high reaction temperature where the mobility of the gel phase increases, the reactant molecules move easily into the microparticle and the

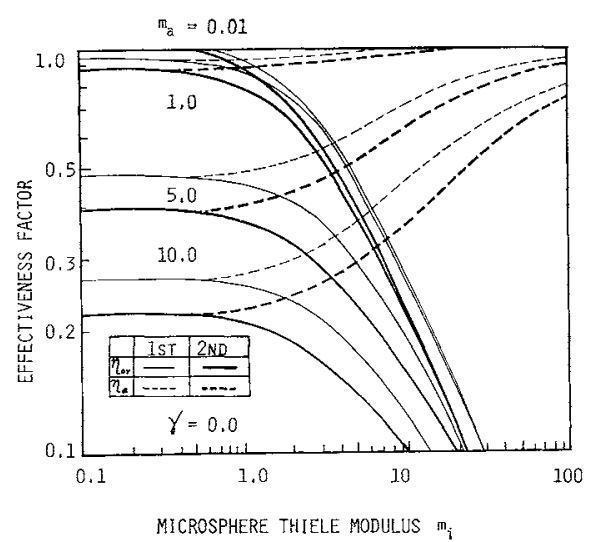

Fig. 4 Effectiveness factor vs. $\boldsymbol{m}_{i}$ and $\boldsymbol{m}_{a} ; \gamma=0$

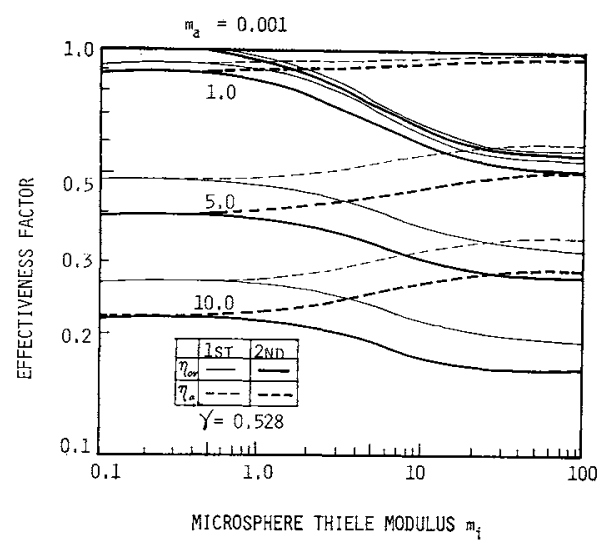

Fig. 5 Effectiveness factor vs. $m_{i}$ and $m_{a} ; \gamma=$ 0.528

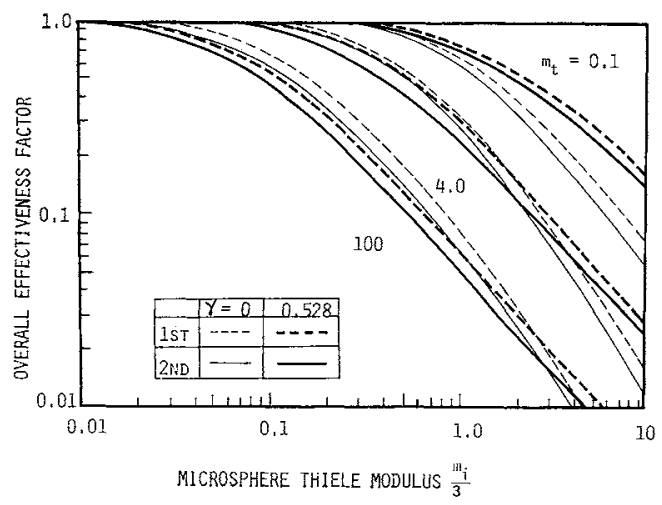

Fig. 6 Overall effectiveness factor

microsphere effectiveness factor becomes unity. These phenomena can be written in the following expression for $\eta_{a}=1$,

$$
\eta_{o v}=\left[\begin{array}{rrl}
\gamma & \left(\bar{\eta}_{i}=0\right) ; & \text { low-temperature range } \\
\gamma+(1-\gamma) \bar{\eta}_{i} & \\
\left(0<\bar{\eta}_{i}<1\right) ; & \text { intermediate range } \\
1 & \left(\bar{\eta}_{i}=1\right) ; & \text { high-temperature range }
\end{array}\right.
$$

and are illustrated as an Arrhenius plot in Fig. 7.

B) Sucrose inversion was performed in a batch reactor, varying the particle sizes and the reaction 


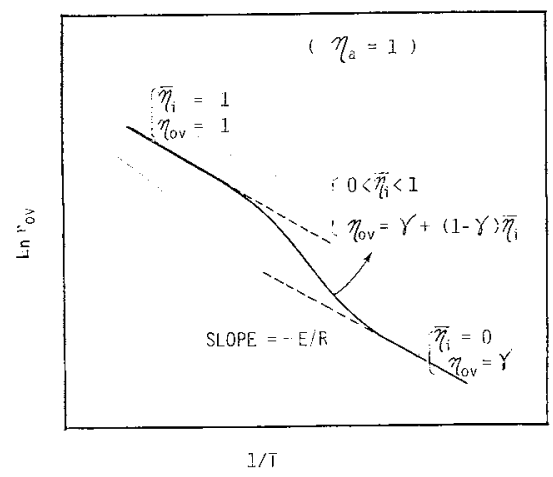

Fig. 7 Arrhenius plot of reaction rate for macroreticular resin catalyst with large $\gamma$

temperatures by using also Amberlyst 15 and Amberlyst $\mathrm{XN}-1010^{5}$. In this case the overall initial reaction rate $r_{o v}$ is written as

$$
\begin{aligned}
r_{o v} & =k C_{o} \eta_{o v} \\
& =k C_{o}\left[\gamma+(1-\gamma) \bar{\eta}_{i}\right] \eta_{a} \\
& =k_{a p p} C_{o} \eta_{a}
\end{aligned}
$$

where $k_{a p p}$ is the apparent reaction rate constant, or

$$
k_{a p p}=k\left[\gamma+(1-\gamma) \overline{\eta_{i}}\right]
$$

It is reasonable to assume for isothermal conditions that as the intrinsic reaction rate constant $k$, the radius $R_{i}$ of the microparticle and the diffusivity $D_{i}$ in the microparticle are constant and independent of the resin sizes, the apparent reaction rate constant $k_{a, p p}$ is also constant and independent of the sizes of resin particles. The values of $D_{a}$ and $k_{a p p}$ were estimated from measurements of the overall reaction rate for given values of particle size and catalyst quantity, and the correlation between experimental data and the present model for $\eta_{a}$ was found to be excellent.

\section{Conclusion}

Macroreticular ion exchange resin catalyst has been envisaged as two phases: the microspheres and the pore space formed between them. For an $n$-th-order irreversible reaction at steady state the overall effectiveness factor $\eta_{o v}$ can be expressed in terms of the average micro-effectiveness factor $\bar{\eta}_{i}$, macro-effectiveness factor $\eta_{a}$ and the fraction $\gamma$ of active sites located on the pore walls, or

$$
\eta_{o v}=\left[\gamma+(1-\gamma) \tilde{\eta}_{i}\right] \eta_{a}
$$

and has been evaluated analytically for a first-order reaction and numerically for a second-order reaction.

In addition to the case of a macroreticular resin catalyst with some fraction of the active sites on the pore walls, two other possible cases of heterogeneous catalysts can be explained by the present model in terms of different ranges of $\gamma$, i.e. a bidisperse porous catalyst for $\gamma=0$ and a catalyst with uniform pore size for $\gamma=1$.

\section{Acknowledgment}

This work has been partially supported by the grants from the Korea Science and Engineering Foundation.

(Presented at the 2nd World Congress of Chem. Eng., Montreal, Canada, Oct. 4-9, 1981).

\section{Nomenclature}

$C=$ concentration; $C_{a}$, in the pore; $C_{i}$ in the

$\begin{aligned} & \text { microsphere; } C_{o} \text { in the bulk } \\ D \quad= & \text { effective diffusivity; } D_{a}, \text { in the pore; }\end{aligned}$ $D_{i}$, in the microsphere $\left[\mathrm{cm}^{2} / \mathrm{sec}\right]$

$e=\quad$ total capacity of a resin particle [equivalent]

$k=$ reaction rate constant $\quad\left[\mathrm{cm}^{3 n} / \mathrm{sec} \cdot\right.$ eq. $\left.\mathrm{mol}^{n-1}\right]$

$M \quad=$ modified Thiele modulus defined by Eq. (15)

$m \quad=$ dimensionless variable; $m_{i}$, defined by Eq. (2); $m_{a}$, defined by Eq. (3); $m_{t}$ defined by Eq. (7)

$n \quad=$ reaction order

$n^{\prime} \quad=$ number of microspheres in a resin particle

$R \quad=$ radius; $R_{a}$, of a resin particle; $R_{i}$, of a microsphere

[cm]

$=$ radial position from the center; $r_{a}$, of a resin particle; $r_{i}$, of a microsphere [cm]

$=$ overall initail reaction rate

$=$ intrinsic reaction rate

$=$ volume, $V_{a}$, of a resin particle; $V_{i}$, of a microsphere

$=$ dimensionless distance from the center; $x_{a}$, for a resin particle, defined by Eq. (1c): $x_{i}$, for a microparticle, defined by Eq. (1d)

$y=$ dimensionless concentration; $y_{a}$, in a resin particle, defined by Eq. (1a); $y_{i}$, in a microsphere, defined by Eq. (1b)

$\gamma \quad=$ fraction of active sites distributed over the pore walls

$=$ porosity

$=$ effectiveness factor; $\eta_{i}$, for a microsphere, $\eta_{a}$, for a resin particle; $\eta_{o v}$ for overall resin

\section{Literature Cited}

1) Aris, R.: "The Mathematical Theory of Diffusion and Reaction in Permeable Catalysts", Vols. I and II, Oxford Univ. Press, London, 1975.

2) Carberry, J. J.: AIChEJ., 8, 557 (1962).

3) Frisch, N. W.: Chem. Eng. Sci., 17, 735 (1962).

4) Mingle, J. O. and J. M. Smith: AIChE J, 7, 243, (1961).

5) Oh, I. H.: "Sucrose Inversion Catalyzed by Macroreticular Ion Exchange Resins," M. S. Thesis (1981), Korea Advanced Institute of Science and Technology. To be published.

6) Ors, N. and T. Dogu: AIChEJ., 25, 723 (1979).

7) Ruckenstein, E., A. S. Vaidyanathan, and G. R. Youngquist: Chem. Eng. Sci., 26, 1305 (1971).

8) Suh, S. S. and S. K. Ihm: J. Korean Inst. Chem. Eng., 18(6) 461 (1980).

9) Tartarelli, R., S. Cioni, and M. Capovani: J. Catalysis, 18, 212 (1970). 